\title{
Transverse Tarsal Joint
}

National Cancer Institute

\section{Source}

National Cancer Institute. Transverse Tarsal Joint. NCI Thesaurus. Code C102354.

A combination of syndesmosis and synovial joints formed by the articulation of the talus with the navicular and the calcaneus with the cuboid. 